\title{
Urinary Tract Infection in a Preterm Neonate Caused by Lactococcus lactis
}

\author{
Brandi Newby and Kuppuchipalayam K Ramesh
}

\section{INTRODUCTION}

$\mathrm{B}_{\mathrm{n}}^{\mathrm{a}}$ acteria of the genus Lactococcus are commonly used in manufacturing dairy products. ${ }^{1}$ This genus of bacteria is also being investigated for use in the biotechnology industry, as a delivery system for vaccines and other therapies. ${ }^{1}$ It is rarely considered pathogenic in humans, although several cases of clinically significant Lactococcus infection have been reported. In adults, these infections have included endocarditis, liver abscess, peritonitis, pneumonitis, spondylodiscitis, septic arthritis, osteomyelitis, sepsis, deep neck infection, and brain abscess. ${ }^{2-4}$ Among pediatric patients, a few cases have been reported, including brain abscess in a 19-month-old child, ${ }^{4}$ catheter-related bacteremia in a 9-month-old infant, ${ }^{5}$ and meningitis and septicemia in a 16 -day-old neonate. ${ }^{3}$ This article describes a preterm neonate who experienced a urinary tract infection caused by Lactococcus lactis.

\section{CASE REPORT}

A male infant was born vaginally at 31 weeks gestational age, with a birth weight of $1557 \mathrm{~g}$.* The birth, to a 33-year-old woman, followed premature rupture of membranes and onset of preterm labour. The infant's Apgar scores were 7 at $1 \mathrm{~min}$ and 9 at $5 \mathrm{~min}$. For the first 13 days, the infant followed a typical course in the neonatal intensive care unit (NICU). He received $48 \mathrm{~h}$ of ampicillin and gentamicin therapy starting at the time of birth, intermittent phototherapy until day 6 , and total parenteral nutrition until day 8, when full feeding, via nasogastric tube with expressed breast milk and human milk fortifier, was achieved. The infant had occasional apnea, bradycardia, and desaturation requiring only mild stimulation or change of position to resolve. The only remarkable laboratory results in the first 13 days were elevated serum creatinine, which peaked at $106 \mu \mathrm{mol} / \mathrm{L}$ (normal range $10-90 \mu \mathrm{mol} / \mathrm{L}$ ) on day 2 , and elevated blood urea nitrogen, which peaked at $24 \mathrm{mmoL} / \mathrm{L}$ (normal range $2-9 \mathrm{mmol} / \mathrm{L}$ ) on

\footnotetext{
*The patient's guardian provided informed consent for publication of this case report.
}

day 4. Renal ultrasonography was performed on day 6 because of the persistently high serum creatinine and blood urea nitrogen levels. The imaging showed moderate bilateral hydronephrosis, hydroureter, echogenicity of the kidneys, and decreased corticomedullary differentiation in the left kidney. The serum creatinine and blood urea nitrogen declined slowly over the course of the admission, and on day 39 their values were within the normal range $(40 \mu \mathrm{mol} / \mathrm{L}$ and $5 \mathrm{mmol} / \mathrm{L}$, respectively).

On day 14, the infant became lethargic, there was an increase in the frequency and severity of apnea, bradycardia, and desaturations requiring oxygen, and the infant's temperature declined to $36.3^{\circ} \mathrm{C}$, which necessitated an increase in incubator temperature of $1.5^{\circ} \mathrm{C}$ to maintain normothermia. Feeds were held, samples of blood and urine were obtained for culture, and the infant was started on vancomycin $12 \mathrm{mg} / \mathrm{kg}$ IV q $8 \mathrm{~h}$ and cefotaxime $50 \mathrm{mg} / \mathrm{kg}$ IV q8h. The complete blood count was normal, but C-reactive protein was elevated, at $41 \mathrm{mg} / \mathrm{L}$ (normal range less than $10 \mathrm{mg} / \mathrm{L}$ ). The urinalysis showed 5 to 10 white blood cells per high-power field, and the urine was positive for leukocyte esterase. Within $24 \mathrm{~h}$ after antibiotics were started, the infant was weaned to room air, and the frequency of apnea, bradycardia, and desaturations returned to baseline frequency. Blood culture showed no growth. Urine culture, for which the sample had been collected via in-and-out catheterization, was positive for Lactococcus, with more than 100 mega-colony-forming units per litre. Growth of L. lactis was confirmed using $16 S$ ribosomal RNA gene sequence analysis. Initial testing showed that the bacterial strain was susceptible to penicillin, cefotaxime, vancomycin, and meropenem.

On day 18, vancomycin and cefotaxime were discontinued, and penicillin 40000 units/kg IV q8h was started. However, the penicillin was discontinued and cefotaxime restarted the following day when a revised susceptibility report indicated intermediate resistance of the Lactococcus strain to penicillin. To ensure clearance of the bacteria, a repeat sample for urine culture was collected on day 19; this sample did not grow any bacteria. IV antibiotics were discontinued after 7 days of therapy. Voiding cystourethrography was performed on day 21, which showed grade 5 reflux on 
the left side and grade 2 reflux on the right side. Therefore, amoxicillin was started for urinary tract infection prophylaxis following completion of the IV antibiotics.

Potential sources of the Lactococcus infection were considered. Although the mother was asymptomatic, her breast milk was sent for culture on day 19 and grew L. lactis, grade 3+ (usual range: no organisms identified to grade $4+$ ). The mother completed a 7-day course of empiric cephalexin, and subsequent testing of her breast milk showed no growth of Lactococcus. She was questioned about potential sources of Lactococcus exposure, but no obvious sources, such as consumption of raw or unpasteurized dairy products or proximity to farm animals, were identified.

Additional analysis of antibiotic susceptibility of the Lactococcus cultured from the infant's urine was requested, for potential treatment of future infection; the results indicated resistance to cefixime, amoxicillin, and trimethoprim; intermediate resistance to cephalexin; and susceptibility to amoxicillinclavulanate. The remainder of the infant's clinical course in the NICU was uneventful, without relapse of the infection. The infant was discharged after 42 days in hospital, in accordance with the institution's criteria for discharge of premature infants.

\section{DISCUSSION}

To the best of the authors' knowledge, this is the first reported case of urinary tract infection caused by $L$. lactis. Urinary tract infections occur in about $1 \%$ of term neonates, with a higher prevalence among preterm neonates. ${ }^{6}$ Bacteria that commonly cause urinary tract infections, such as Escherichia coli, Klebsiella, and Enterobacter, travel from the gastrointestinal tract and anogenital area into the urinary tract. ${ }^{7}$ In this case, L. lactis was thought to have come from the gastrointestinal tract after ingestion of the mother's breast milk.

Organisms generally considered to be contaminants or nonpathogenic in the immunocompetent population, such as Lactococcus, can cause significant clinical infection in immunocompromised individuals. The questionable pathogenicity of these organisms can lead to delays in identifying the organism, reporting antibiotic susceptibility, and initiating appropriate antibiotic therapy. In the case reported here, the infant exhibited clinical correlates of infection, including hypothermia, lethargy, and an increase in apnea, bradycardia, and desaturation episodes, which led the authors to request analysis of antibiotic susceptibility, as well as testing of the mother's breast milk. Given that premature neonates are at increased risk of infection due to the immaturity of their immune systems, any organism identified in a symptomatic neonate should be considered a pathogen.

In this case, the antibiotic susceptibility of the strain was unexpected. Lactococcus is a genus of facultative anaerobic catalase-negative, gram-positive cocci previously classified as Streptococcus. ${ }^{2,4}$ Other strains of Lactococcus have been reported to be susceptible to penicillin, cephalosporins, vancomycin, meropenem, and macrolides. ${ }^{2-5}$ However, in this case, the strain was resistant or intermediately resistant to several antibiotics to which it was expected to be susceptible, including penicillin, amoxicillin, cephalexin, cefixime, and trimethoprim.

Breast milk has many infection-related benefits, including association with a lower risk of urinary tract infection in neonates. ${ }^{7}$ Breast milk is rarely the cause of infections in neonates, but should be considered a possible source for unusual, difficultto-eradicate, or recurrent infections. In this case, the mother's breast milk was positive for $L$. lactis and was therefore considered to be the source of the infant's infection. Despite the resistance pattern found in vitro, Lactococcus was not detected in the mother's milk following a 7-day course of cephalexin. It is unknown how the mother acquired L. lactis, as she had no known exposure to cattle, the common host for Lactococcus, or contaminated dairy products. Lactococcus has occasionally been identified as a component of the normal flora in humans ${ }^{4}$; this may have been a possibility for this mother, as the organism was not pathogenic for her.

This case adds to the documented evidence that $L$. lactis can be pathogenic in neonates. It also highlights the importance of analyzing antibiotic susceptibility for uncommon pathogens, as susceptibility patterns can vary.

\section{References}

1. Bahey-El-Din M, Gahan CG. Lactococcus lactis: from the dairy industry to antigen and therapeutic protein delivery. Discov Med. 2010;9(48):455-61.

2. Chan JFW, Woo PCY, Teng JLL, Lau SKP, Leung SSM, Tam FCC, et al. Primary infective spondylodiscitis caused by Lactococcus garvieae and a review of human L. garvieae infections. Infection. 2011;39(3):259-64.

3. Uchida Y, Morita H, Adachi S, Asano T, Taga T, Kondo N. Bacterial meningitis and septicemia of neonate due to Lactococcus lactis. Pediatr Int. 2011;53(1): 119-20.

4. Topçu Y, Akıncı G, Bayram E, Hız S, Türkmen M. Brain abscess caused by Lactococcus lactis cremoris in a child. Eur J Pediatr. 2011;170(12):1603-5.

5. Glikman D, Sprecher H, Chernokozinsky A, Weintraub Z. Lactococcus lactis catheter-related bacteremia in an infant. Infection. 2010;38(2):145-6.

6. Bauer S, Eliakim A, Pomeranz A, Regev R, Litmanovits I, Arnon S, et al. Urinary tract infection in very low birth weight preterm infants. Pediatr Infect Dis J. 2003;22(5):426-30.

7. Levy I, Comarsca J, Davidovits M, Klinger G, Sirota L, Linder N. Urinary tract infection in preterm infants: the protective role of breastfeeding. Pediatr Nephrol. 2009;24(3):527-31.

Brandi Newby, BScPharm, ACPR, is Clinical Supervisor of the Neonatal and Pediatric Pharmacy, Surrey Memorial Hospital, Surrey, British Columbia

Kuppuchipalayam K Ramesh, MB, DCH, MRCP(Ire.), FRCPC, is a Consultant Neonatologist with the Department of Neonatology, Surrey Memorial Hospital, Surrey, British Columbia.

Competing interests: None declared.

Address correspondence to:

Brandi Newby

Neonatal and Pediatric Pharmacy, Room 2-602

Critical Care Tower

Surrey Memorial Hospital

13750 96th Ave

Surrey BC V3V 1 Z2

e-mail: Brandi.newby@fraserhealth.ca 